\title{
CARACTERÍSTICAS ANTROPOMÉTRICAS Y DE POTENCIA MUSCULAR EN FUTBOLISTAS COSTARRICENSES ENTRE LOS 15 Y 20 AÑ̃S
}

\section{ANTHROPOMETRIC CHARACTERISTICS AND MUSCULAR STRENGTH IN COSTA RICAN FOOTBALL PLAYERS BETWEEN 15 AND 20 YEARS}

\author{
Mario Esteban Serrano Sanabria \\ Greivin Javier Mora Poveda \\ Braulio Sánchez Ureña (0000-0001-8791-6836) 1,2 \\ Juan Carlos Gutiérrez Vargas (0000-0002-0689-6771) \\ Miguel Eduardo Méndez Solano \\ ${ }^{1}$ Centro de Investigación y Diagnóstico en Salud y Deporte, Escuela Ciencias del \\ Movimiento Humano y Calidad de Vida, Universidad Nacional \\ ${ }^{2}$ Programa Ciencias del Ejercicio y la Salud, Escuela Ciencias del Movimiento Humano y \\ Calidad de Vida, Universidad Nacional, Costa Rica \\ ${ }^{3}$ Centro de Investigación y Diagnóstico en Salud y Deporte, Escuela Ciencias del \\ Movimiento Humano y Calidad de Vida, Universidad Nacional \\ estebanss12@gmail.com
}

\section{Resumen}

El propósito del estudio fue comparar las características antropométricas y las capacidades neuromusculares en futbolistas costarricenses según su posición en el terreno de juego. Se evaluó un total de 84 futbolistas de cinco equipos de la división de alto rendimiento del fútbol costarricense con una edad promedio de 17.8 \pm 1.2 años. El porcentaje de grasa se midió mediante siete pliegues cutáneos; la flexibilidad por medio de la prueba de Sit and Reach; la potencia de miembros inferiores mediante los test de Bosco: Squat Jump, Counter Movement Jump e Índice de Fatiga en 30s y se aplicó el test de velocidad en 10 y 25 m. Se encontraron diferencias significativas en el peso según la posición de juego $(\mathrm{F}(3,80)=5.54, \mathrm{p}=0.002)$ : el peso promedio de los porteros es de $76.12 \pm 8.92 \mathrm{~kg}$, significativamente mayor que el de los defensas $(\mathrm{p}=0.01)$, volantes ( $\mathrm{p}$ $=0.03)$ y delanteros $(\mathrm{p}=0.001)$. También se registraron diferencias estadísticamente significativas en la variable talla en el contraste entre puestos $\mathrm{F}(3,80)=6.50, \mathrm{p}=0.001$ : la talla promedio de los porteros es de 1.81 $\pm 0.05 \mathrm{~m}$ significativamente mayor en comparación a volantes $1.73 \pm 0.5 \mathrm{~m}(\mathrm{p}=0.003)$ y delanteros $1.72 \pm$ $0.63 \mathrm{~m}(\mathrm{p}=0.001)$. En el índice de fatiga se presentaron diferencias significativas. En las demás variables no se encontraron diferencias significativas. Los resultados de este estudio concuerdan con la literatura existente para futbolistas de las mismas categorías. De las comparaciones realizadas entre cada uno de los puestos, en las variables estudiadas no se evidencia la especificidad físico-funcional.

Palabras clave: antropometría, fútbol, jóvenes, capacidad muscular

\begin{abstract}
The objective of this research was to compare the anthropometric characteristics and neuromuscular capabilities of Costa Rican soccer players based on their positions on the soccer field. A total of 84 soccer pla-
\end{abstract}


yers, average ages $17.8 \pm 1.2$, from five Costa Rican high performance division teams were evaluated. Fat percentage was measured by the sum of seven skin folds, flexibility by the Sit and Reach test, the power of lower limps by Bosco's Squat Jump and Countermovement Jump tests, and the Fatigue Index in 30 seconds. Lastly, a speed test was applied for 10 and 25 meters. Significant differences were found in the players' weight according to their position on the soccer field $F_{(3-80)}=5.54, p=0.002$. For instance, the average weight of goalkeepers is $76.12 \pm 8.92 \mathrm{~kg}$, which is significantly higher than the weight of defenders $(p=0.01)$, midfielders $(p=0.03)$, and forwards $(p=0.001)$. Statistically significant differences were also recorded on the height variable among positions $F(3-80)=6.50, p=0.001$. For instance, goalkeepers' average height was 1.81 $\pm 0.05 \mathrm{~m}$, which was significantly higher than that of midfielders $1.73 \pm 0.5 \mathrm{~m}(p=0.003)$ and forwards 1.72 $\pm 0.63 \mathrm{~m}(p=0.001)$. Significant differences were also found in the fatigue index, $F(3-80)=3.22, p=0.02$, between goalkeepers and midfielders. No statistically significant differences were found in the rest of the variables. The results found in this study agree with the existing literature for soccer players in the same categories. There is no evidence of the physical functional specificity on the studied variables regarding comparisons between positions.

Key word: anthropometrics, soccer, young, muscular capacity

\section{Introducción}

La evaluación fisiológica en el deporte es de gran importancia en la consecución de mejores resultados en la forma deportiva óptima (Kalentić, Golik, Doder, Sudarov y Jovančević, 2012; Mariano, de Arruda y Pascoal, 2010), en especial cuando la tecnología actualmente permite estudiar aún más a cada deportista según su especialidad y de acuerdo con sus necesidades. Las evaluaciones se utilizan para tener un mayor control y medir el progreso de los deportistas según la etapa de la temporada en la que se encuentra (Portella, De Arruda, y Cossio, 2011; Castellanos, Sánchez, González y Carvajal, 2011).

En los últimos años, las evaluaciones fisiológicas igualmente han adquirido gran relevancia en la preparación del futbolista con el fin de mejorar y maximizar sus capacidades. Al existir mayor cantidad de estudios de diversas evaluaciones fisiológicas en futbolistas (Calahorro, Zagalaz, Lara y Torres, 2012; Hernández-Mosqueira et al., 2013; Prieto y García, 2013; Sánchez, Pérez, Coco, Ruiz y Marín, 2013; Tissue, 2014; Zubeldía, 2010) es posible tener una mayor cantidad de datos para medir a los jugadores en distintas pruebas de aptitud física que permitan mejorar sus capacidades físicas y fisiológicas.

También, en busca de mejores resultados y en la detección de talentos en edades de formación deportiva, se encuentran las evaluaciones antropométricas. Con base en estas se puede establecer entrenamientos específicos para los deportistas, según su talla, peso, porcentaje de grasa, entre otros (Gil y Verdoy, 2011; Ramos, Aguirre y Gutiérrez, 2015). Al respecto, se han reportado numerosos estudios en relación con evaluaciones antropométricas en busca de mejorar las capacidades de los futbolistas en edades de formación (Benítez, Da Silva, Muñoz, Morente y Guillén, 2015; Gil y Verdoy, 2011; Jorquera, Rodríguez, Torrealba y Barraza, 2012; Mosqueira et al., 2013; Pazo, López y Fradua, 2012; Ramos, Aguirre y Gutiérrez, 2015; Sinivas et al. 2015). 
En el fútbol, un jugador está comprometido en muchas acciones exigentes de alta demanda energética (Salazar et al., 2017), por lo que será demandado físicamente en partidos y entrenamientos. Debido a esto, la evaluación fisiológica permite tener un mayor control del esfuerzo que el futbolista debe realizar al competir, para así poderlo capacitar de la mejor manera a tolerar las cargas físicas y fisiológicas a las que se vea sometido (Ruiz, Romero, Fernández, Morcillo y Mariscal, 2015; Salazar et al., 2017). De esta manera, las evaluaciones físicas son un medio óptimo para el control y la evaluación de deportistas, ya que se evalúan cuantitativa y cualitativa aspectos físicos, técnicos y tácticos necesarios para el deporte, en este caso para el fútbol (García, Ruiz, y Latorre, 2015; González, Campos, y Romero, 2014). Dichas evaluaciones brindan datos significativos para que los cuerpos técnicos puedan realizar una mejor planificación individual y grupal a partir de los datos de cada prueba efectuada (González et al., 2014; Prieto y García, 2013).

Entre los tipos de puestos desempeñados por los jugadores de fútbol se espera que existan diferencias estadísticamente significativas en sus características fisiológicas y antropométricas, debido a las demandas que exige cada uno (Calahorro et al., 2012; Sánchez, Araya, Blanco y Crespo, 2016; Sánchez, Salas, Blanco y Araya, 2011). Por esto, los datos obtenidos de estudios sobre características antropométricas y fisiológicas dan la oportunidad a los entrenadores de realizar entrenamientos específicos para las exigencias que influyen en cada jugador según su puesto (Sánchez et al., 2011). Al encontrar diferencias específicas tanto en los elementos antropométricos y fisiológicos en cada puesto, se deben realizar trabajos precisos según la posición del jugador, que serán determinantes para poder alcanzar el máximo rendimiento posible (Fernández, Kazarez, Agazzi y Albin, 2014).

A nivel internacional existen parámetros que se confrontan en diversas partes del mundo como punto de partida para las distintas pruebas antropométricas y fisiológicas a realizar. Todo esto posibilita comparaciones de características antropométricas y fisiológicas con mayor validez de acuerdo con el país en que se efectuaron las evaluaciones (Cornejo, 2016; Sánchez et al., 2011), de aquí la importancia de la determinación de parámetros específicos en cada región. Sobre este tipo de evaluaciones se han elaborado numerosos estudios en otras regiones de América (Cardenal y Quintero, 2015; Fernández et al., 2014; Jorquera, Rodríguez, Torrealba y Barraza, 2012; Toro y Peláez, 2014,) y en Europa (Bidaurrazaga, Lekue, Amado, Santos y Gil, 2015; Carrasco, Calahorro, Lara y Torres, 2014; Iglesias, Grijota, Crespo, Llerena y Muñoz, 2013; Muñoz, 2015). No obstante, en la región centroamericana son escasos los estudios realizados sobre evaluaciones fisiológicas y antropométricas en edades comprendidas entre los 15 y 20 años.

Morgans, Orme, Anderson y Drust (2014) afirman que la poca planificación en general, en divisiones inferiores, limita el desarrollo de jugadores de fútbol de buen nivel. Esta situación afecta a Costa Rica y deja en rezago a sus jugadores en comparación con países más avanzados. Respecto a esto, la valoración fisiológica toma un valor destacable a la hora de detectar talentos y de perfeccionar a cada futbolista en edades sensibles de mejoramiento (García, Ruiz, y Latorre, 2015). Al no existir un trabajo que haya comparado las características antropométricas 
y capacidades neuromusculares en edades de 15 a 20 años según su posición en el campo de juego en Costa Rica y al encontrar solamente una investigación de este tipo en el país a nivel élite (Sánchez et al., 2011), el propósito del artículo es comparar las características antropométricas y capacidades neuromusculares en futbolistas costarricenses entre los 15 y 20 años según su posición en el terreno de juego.

\section{Metodología}

\section{Tipo de estudio}

Comparativo y de corte transversal.

\section{Participantes}

Participaron un total de 84 futbolistas pertenecientes a cinco equipos de la división de alto rendimiento del fútbol costarricense, con edad promedio de 17. $8 \pm 1.23$ años, los cuales fueron categorizados según su posición de juego en porteros $(11 \%, n=9)$, defensas $(34 \%, n=29)$, mediocampistas $(30 \%, n=25)$ y delanteros $(25 \%, n=21)$. Los jugadores son procedentes de las provincias San José, Cartago y Limón, y fueron elegidos mediante un muestreo a conveniencia de grupos naturalmente constituidos.

\section{Instrumentos y materiales}

La medición del peso, estatura y porcentaje de grasa se realizó según el protocolo del American Collegue of Sport Medicine (ACSM, 2014). La estatura se realizó respetando el plano de Frankfort, mediante un estadiómetro marca "Tanita". El peso fue evaluado con una báscula, también de marca "Tanita”, modelo HD-313, con una precisión de $\pm 0,1 \mathrm{~kg}$. A su vez, el porcentaje de grasa fue obtenido por medio de pliegues cutáneos, utilizando el protocolo de siete pliegues (pectoral, axilar, subescapular, tríceps, suprailiaco, abdominal y muslo). Esta medición se realizó con un plicómetro marca "Lange", con una precisión de 0,2 mm y una sensibilidad de $1 \mathrm{~mm}$, y se efectuaron tres mediciones de cada uno de los pliegues del lado derecho del cuerpo de los sujetos evaluados.

Las capacidades neuromusculares (fuerza explosiva, resistencia a la fuerza veloz e índice de fatiga) se valoraron mediante los test del Squat Jump, el Countermovement Jump y el test de saltos continuos en treinta segundos, todos mediante el protocolo del test de Bosco, el cual tiene una validez de $r=0.95$ (Bosco, Luhtanen y Komi, 1983). Para la recolección de los datos y la realización de las pruebas fue utilizada una plataforma de fuerza de movimientos marca "Newtest". 
El test de velocidad se realizó en 25 metros con salida en el metro cero y con una medición del tiempo parcial (Lap) en el metro 10, estas mediciones fueron tomadas mediante fotocensores de movimientos marca "Newtest".

Para evaluar la flexibilidad de la parte baja de la espalda, los extensores de la cadera y los músculos flexores de la rodilla, se utilizó la prueba Sit and Reach, en la que se empleó un banco sueco o Cajón de Wells.

\section{Procedimiento}

Los participantes recibieron información de las distintas pruebas antes de su realización, las cuales constituyen parte del protocolo de evaluaciones del presente estudio. En primera instancia se contó con el consentimiento de los jugadores, respetándose con ello las normas de Helsinki, y posteriormente se solicitó autorización a cada uno de los clubes participantes para la publicación de los datos. Se explicó a los sujetos el proceder de cada una de las mediciones antes de su ejecución y el orden a seguir indicado por el Laboratorio de Evaluación Fisiológica de la Universidad Nacional, Costa Rica. Los equipos realizaron las evaluaciones en la misma sucesión, los sujetos ejecutaron cada una de las valoraciones de forma individual y las pruebas fueron realizadas en condiciones ambientales controladas a $23^{\circ} \mathrm{C}$ en el laboratorio. En el caso de las pruebas de velocidad, estas se realizaron en la misma cancha de fútbol bajo condiciones ambientales similares, los días viernes durante cinco semanas consecutivas, iniciando a las 9:00 a.m.; esto fue igual para todos los equipos.

Se determinó primeramente el peso y la talla, a lo que le siguió la medición por pliegues cutáneos, posterior a un calentamiento de 15 minutos con movimientos articulares y de flexibilidad para preparar de buena manera a los sujetos de evaluación. Luego se realizó la prueba de flexibilidad, la cual consiste en que el sujeto debe sentarse en el suelo con las piernas juntas y extendidas hacia delante, sus pies deben estar pegados a la caja de medición y los brazos y manos extendidos hacia delante; una vez que se esté en la posición inicial de la prueba, el sujeto debe flexionar el tronco hacia delante, empujando con ambas manos, y mantener esta posición unos segundos para que el evaluador pueda ver hasta dónde llega la marca del evaluado. Se efectuaron tres intentos por sujeto y el mejor de esos tres intentos fue tomado para efectos de la evaluación.

Seguidamente se aplicaron las pruebas neuromusculares. La primera, la prueba Squat Jump (SJ), mide la fuerza explosiva y consiste en la realización de un salto vertical máximo partiendo de la posición de flexión de piernas de $90^{\circ}$, sin ningún tipo de rebote o contramovimiento, y en el cual los miembros superiores tampoco intervienen en el salto puesto que las manos deben permanecer en la cadera desde la posición inicial hasta la finalización de salto. El sujeto en la fase de vuelo debe mantener el cuerpo erguido, las piernas extendidas y pies en flexión plantar. Además, debe efectuar la caída en el mismo lugar de inicio, con los brazos fijados en la cadera. La segunda prueba, Counter Movement Jump (CMJ), evalúa la resistencia a la fuerza. Esta se realiza con un movimiento rápido de flexo-extensión de las rodillas, formando 
durante la bajada un ángulo de $90^{\circ}$ con las rodillas, e inmediatamente se realiza un salto vertical máximo. Posterior a estas dos pruebas, se aplicó la prueba del índice de fatiga en 30 segundos consecutivos. Su estimación se realizó calculando el promedio de altura de los últimos cinco saltos dividido entre el promedio de altura de los primeros cinco, esto para estimar la capacidad anaeróbica láctica. Se finaliza con la prueba de velocidad en 25 metros. Todas las mediciones se realizaron a máxima intensidad.

\section{Análisis estadístico}

Se utilizó el paquete estadístico para las ciencias sociales SPSS (v. 18.0, SPSS, Inc., Chicago, IL.). Se calcularon estadísticas descriptivas (promedio, desviación estándar, mínimos y máximos) para todos los datos. La normalidad de los datos fue evaluada por medio del Kolmogorov Smirnov Test. La homogeneidad de las varianzas se realizó mediante el Test de Levene. Se utilizó el análisis de varianza (ANOVA) de una vía con Bonferroni como técnica post hoc para determinar diferencias entre puestos. El nivel de significancia utilizado correspondió a $p<0.05$.

\section{Resultados}

En la tabla 1 se muestra la estadística descriptiva (promedios, desviación estándar, mínimo y máximo) en términos generales para cada una de las variables estudiadas en la totalidad de la muestra.

Tabla 1

Estadística descriptiva de las variables investigadas (promedios, DS, valores mínimos y máximos)

\begin{tabular}{lllll}
\hline Variables & Promedio & DS & Mínimo & Máximo \\
\hline Peso $(\mathrm{kg})$ & 67.91 & 7.63 & 51.00 & 89.20 \\
\hline Edad $($ años $)$ & 17.81 & 1.24 & 15.00 & 20.00 \\
\hline Talla $(\mathrm{cm})$ & 174 & 0.07 & 156 & 190 \\
\hline \% Grasa & 7.08 & 2.91 & 2.79 & 17.80 \\
\hline Flexibilidad $(\mathrm{cm})$ & 40.51 & 7.31 & 21.00 & 60.00 \\
\hline Squat Jump $(\mathrm{cm})$ & 30.21 & 3.47 & 19.00 & 37.00 \\
\hline TV SJ $(\mathrm{ms})$ & 495.36 & 28.71 & 398.00 & 548.00 \\
\hline CMJ $(\mathrm{cm})$ & 38.59 & 4.52 & 29.00 & 52.00 \\
\hline TV CMJ $(\mathrm{ms})$ & 559.65 & 32.81 & 486.00 & 651.00 \\
\hline Índ. Fatiga 30 s & 0.83 & 0.09 & 0.67 & 1.14 \\
\hline Vel. $10 \mathrm{~m} \mathrm{(m/s)}$ & 5.43 & 0.30 & 4.78 & 6.05 \\
\hline Vel. $25 \mathrm{~m} \mathrm{(m/s)}$ & 6.65 & 0.32 & 5.95 & 7.44 \\
\hline
\end{tabular}

Nota: SJ: Squat Jump, CMJ: countermovement jump, TV: tiempo de vuelo, Índ. Fatiga: índice de fatiga, Vel. $10 \mathrm{~m}$ : velocidad en 10 metros, Vel. 25 m: velocidad en 25 metros 
En la tabla 2 se muestran los resultados de la comparación de las características antropométricas según la posición de juego.

Tabla 2

Comparación de medidas antropométricas por posición de juego

\begin{tabular}{llcccccc}
\hline \multirow{2}{*}{ PUESTO } & \multirow{2}{*}{$\mathbf{n}$} & \multicolumn{2}{c}{ Peso $(\mathbf{k g})$} & \multicolumn{2}{c}{ Talla $(\mathbf{c m})$} & \multicolumn{2}{c}{ \% grasa } \\
\cline { 3 - 8 } & & Media & DS & Media & DS & Media & DS \\
\hline Porteros & 9 & $76.12^{\mathrm{a}}$ & 8.92 & $181^{\mathrm{a}}$ & 0.05 & 8.68 & 3.38 \\
\hline Defensas & 29 & $67.38^{\mathrm{b}}$ & 7.71 & 175 & 0.07 & 6.23 & 2.57 \\
\hline Medios & 25 & $68.25^{\mathrm{b}}$ & 7.1 & $173^{\mathrm{b}}$ & 0.5 & 8.15 & 3.3 \\
\hline Delanteros & 21 & $64.71^{\mathrm{b}}$ & 1.72 & $172^{\mathrm{b}}$ & 0.63 & 6.29 & 1.99 \\
\hline
\end{tabular}

Nota: X: promedio, DS: desviación estándar. Letras diferentes simbolizan diferencias estadísticamente significati$\operatorname{vas}(p<0.05)$.

Se observan diferencias estadísticamente significativas en la variable peso según posición de juego $F_{(3-80)}=5.54, p=0.002$. Mediante análisis post hoc Bonferroni se identificó que las diferencias fueron entre porteros y las demás posiciones de juego: defensas $(p=0.01)$, volantes $(p=0.03)$ y delanteros $(p=0.001)$.

Con respecto a la talla, también se registraron diferencias estadísticamente significativas entre los puestos $F_{(3-80)}=6.50, p=0.001$. El análisis post hoc mostró que la talla promedio de los porteros $1.81 \pm 0.05 \mathrm{~m}$, fue significativamente mayor que la talla promedio de los volantes $1.73 \pm 0.5 \mathrm{~m}(p=0.003)$ y que la media de los delanteros $1.72 \pm 0.63 \mathrm{~m} .(p=0.001)$. En lo referente al porcentaje de grasa, no se registraron diferencias significativas entre puestos $(p$ $>0.05$ ), obteniendo el mayor porcentaje de grasa los porteros, con $8.68 \%$, y el menor los defensas, con $6.23 \%$.

La tabla 3 muestra las variables neuromusculares, donde se encontró diferencias significativas entre puestos en la variable índice de fatiga en 30 segundos, $F_{(3-80)}=3.22, p=0.02$, pero esta diferencia se encontró únicamente entre los porteros y los volantes $(p=0.02)$.

En cuanto al nivel de flexibilidad, los mejores valores promedio de flexibilidad los mostraron los porteros $43.11 \pm 9.63 \mathrm{~cm}$, seguido por los delanteros $42.38 \pm 5.21 \mathrm{~cm}$, defensas $40.68 \pm 7.04 \mathrm{~cm}$ y volantes $37.80 \pm 7.75 \mathrm{~cm}$, sin embargo, no se registraron diferencias estadísticamente significativas $(p>0.05)$ entre estos puestos.

Para las variables, flexibilidad, CMJ, el SJ y velocidad en 10 y 25 metros no se presentaron diferencias significativas $(p>0.05)$ entre los puestos. 
Tabla 3

Comparación de variables neuromusculares por posición de juego

\begin{tabular}{|c|c|c|c|c|c|c|c|c|c|c|c|c|c|}
\hline \multirow{2}{*}{$\begin{array}{l}\text { PUESTO } \\
N\end{array}$} & & \multicolumn{2}{|c|}{ Flexib. $(\mathrm{cm})$} & \multicolumn{2}{|c|}{$\begin{array}{l}\text { CMJ } \\
(\mathrm{cm}) \\
\end{array}$} & \multicolumn{2}{|c|}{$\begin{array}{c}\text { SJ } \\
(\mathrm{cm})\end{array}$} & \multicolumn{2}{|c|}{$\begin{array}{c}\text { Índice } \\
\text { fatiga }\end{array}$} & \multicolumn{2}{|c|}{ Vel. $10 \mathrm{~m}(\mathrm{~m} / \mathrm{s})$} & \multicolumn{2}{|c|}{$\begin{array}{l}\text { Vel. } 25 \mathrm{~m} \\
(\mathrm{~m} / \mathrm{s})\end{array}$} \\
\hline & & $\mathrm{X}$ & DS & $\mathrm{X}$ & DS & $\mathrm{X}$ & DS & $\mathrm{X}$ & DS & $\mathrm{X}$ & DS & $\mathrm{X}$ & DS \\
\hline Porteros & 9 & 43.11 & 9.63 & 39 & 5.59 & 31.44 & 3.53 & $0.77^{\mathrm{a}}$ & 0.04 & 5.30 & 0.37 & 6.47 & 0.41 \\
\hline Defensas & 29 & 40.68 & 7.04 & 39.82 & 4.89 & 30.86 & 3.43 & 0.82 & 0.07 & 5.47 & 0.30 & 6.63 & 0.31 \\
\hline Volantes & 25 & 37.80 & 7.75 & 36.80 & 4.08 & 29.08 & 3.37 & $0.86^{\mathrm{b}}$ & 0.94 & 5.37 & 0.27 & 6.65 & 0.30 \\
\hline Delanteros & 21 & 42.38 & 5.21 & 38.85 & 3.52 & 30.14 & 3.48 & 0.82 & 0.09 & 5.50 & 0.29 & 6.75 & 0.32 \\
\hline
\end{tabular}

Nota: X: promedio, DS: desviación estándar, Flexib.: flexibilidad, SJ: Squat Jump, CMJ: Countermovement Jump, Ind. Fat.: índice de fatiga, Vel. $10 \mathrm{~m}$ : velocidad 10 metros, Vel. $25 \mathrm{~m}$ : velocidad 25 metros. Letras diferentes simbolizan diferencias significativas $(p<0.05)$.

\section{Discusión}

El propósito del estudio fue comparar las características antropométricas y capacidades neuromusculares en futbolistas costarricenses de entre 15 y 20 años, según su posición en el terreno de juego. Se registraron diferencias significativas en las variables antropométricas peso y talla, pero no en el porcentaje de grasa corporal. A nivel de las variables neuromusculares, se registraron diferencias significativas únicamente en el índice de fatiga, pero no en las variables squat jump, counter movement jump, flexiblidad y los test de velocidad en 10 y 25 metros.

Los porteros obtuvieron peso y talla mayor que los demás puestos, lo que concuerda con estudios al mismo nivel (Calahorro et al., 2012) y con estudios en futbolistas élite (Fernández et al. 2015; Henríquez, Báez, Ramírez y Cañas, 2013; Jorquera et al., 2013; Sánchez et al., 2011). Esta variabilidad se debe a diferencias físico-funcionales del puesto específico desempeñado, debido a las exigencias particulares dentro del deporte (Maureira, Bahamondes, Jesam y López, 2015). Desde que se inicia con la selección de un portero es normal que se utilice el criterio biomecánico de que, a mayor tamaño, el arquero cubrirá con más facilidad el arco, además de que un arquero alto hará percibir mayor seguridad y tendrá mayor autoconfianza sobre todo en balones aéreos (Sánchez et al., 2011).

En cuanto a los porcentajes de grasa, los resultados muestran que los defensores tienen el porcentaje de grasa más bajo $(6.23 \%)$, lo cual difiere con los resultados del estudio realizado por Calahorro et al. (2012), quien encontró que el porcentaje más bajo lo presentan los delanteros (10.47 \%). Los porcentajes más altos se encuentran en los porteros, lo cual concuerda con estudios existentes (Calahorro et al., 2012; Sánchez et al., 2011; Sánchez et al., 2016; Zubeldía, 2010). Al respecto, 
Sánchez et al. (2011) manifiestan que los porteros no son exigidos fisiológica y bioenergéticamente en competencia, ya que estos compitiendo no deben de realizar grandes desplazamientos, por lo que no comprometen su capacidad de consumo máximo de oxígeno $\left(\mathrm{VO}_{2}\right.$ máx.).

Calahorro et al. (2012) presentan resultados en flexibilidad que concuerdan con los obtenidos en el presente estudio. De acuerdo con ellos, los más flexibles son los porteros y los delanteros, lo cual se podría atribuir al rol específico de estos en el fútbol, ya que para rendir de buena manera deben entrenar mucho la agilidad y flexibilidad, los delanteros para esquivar a los defensores rivales y los porteros para poder estirarse cada vez que van por un balón difícil (Ziv y Lidor, 2011; Soarez, Fragoso, Massuça y Barrigas, 2012).

El valor medio de la prueba CMJ es superior al de estudios similares (Carrasco et al, 2014; Frazilli, de Arruda, Mariano y Cossio, 2011), pero menor que el de los resultados presentados por Méndez, Márquez y Castro, (2011). Además, los valores obtenidos concuerdan con los valores obtenidos por Sinivas et al. (2015), pero discrepan con lo presentado por Sánchez et al., (2011), ya que fueron los defensores los que obtuvieron el valor mayor en esta prueba y no los porteros como se reportó en su estudio. Sin embargo, tampoco se alejan mucho de esos resultados, pues los porteros fueron los segundos en este aspecto, lo cual se puede deber a que los defensores y porteros requieren de una capacidad de salto elevada (Le Gall et al., 2010; García y Peña, 2016) para rendir de buena manera en muchas acciones de juego aéreo que se les presentan en entrenamientos y partidos, por lo que al ejercitar más esta cualidad perfeccionan en mayor grado su ciclo de estiramiento-acortamiento muscular.

Los resultados del estudio respecto al índice de fatiga indican que los volantes son los que obtuvieron los índices más bajos, pues al ser estos los que recorren más terreno a lo largo de las competencias tanto en labores ofensivas como defensivas, ejecutando cambios rápidos de ritmo y dirección con periodos cortos de recuperación, son superiores en cuanto a capacidad aeróbica y anaeróbica (Búa, Rodríguez y García, 2013; Sánchez et al., 2011), por ende sobresalen de los otros puestos en cuanto a las mediciones del índice de fatiga. Los porteros, por la especificidad de acciones que realizan en el fútbol, tienden a tener mayores índices de fatiga, ya que en competencias y entrenamientos sus intervenciones son de muy poca duración (unos pocos segundos o centésimas), por lo que no ven comprometida su capacidad anaeróbica láctica (Salazar et al., 2017). Los porteros además fueron los que obtuvieron la mayor velocidad en 10 metros, concordando con lo reportado por Sánchez et al., (2011). Este resultado se atribuye a la superioridad de estos en la variable talla con respecto a los otros puestos, ya que, con zancadas más amplias, a estos se les facilita hacer los recorridos en un menor tiempo (Oliveros, 2015).

\section{Conclusiones}

Se concluye que los resultados encontrados en este estudio concuerdan en su mayoría con la literatura existente en cuanto a características antropométricas y capacidades neuromusculares de futbolistas de las edades estudiadas.

El comportamiento de las variables analizadas en este estudio registró diferencias significativas entre puestos solo en algunos casos, por lo que no se evidencia la especificidad 
físico-funcional de cada uno de los puestos, lo que hace reflexionar sobre la especificidad de los trabajos desarrollados en función de las posiciones de juego de cada futbolista, en particular en los participantes de este estudio.

Finalmente, la información obtenida en el presente estudio constituye un punto de partida importante en el desarrollo de divisiones menores del fútbol costarricense, en vista de que son los primeros datos generados en las variables analizadas en futbolistas jóvenes costarricenses.

\section{Referencias bibliográficas}

American College of Sports Medicine [AMCS] (2014). Guidelines for Exercise Testing and Prescription. (9a Ed.). Philadelphia: Lippincott Willian and Wilkins.

Benítez, J., Da Silva, M., Muñoz, E., Morente, A., Guillén, M. (2015). Capacidades físicas en jugadores de fútbol formativo de un club profesional. Revista Internacional de Medicina y Ciencias y de la Actividad Física del Deporte. 15(58), 289-307. Recuperado de https://doi. org/10.15366/rimcafd2015.58.006

Bidaurrazaga, I., Lekue, J., Amado, M., Santos, J., Gil, S. (2015). Identificación de jóvenes talentos en fútbol: características condicionales, antropométricas y fisiológicas como predictores del rendimiento. Revista Internacional de Ciencias del Deporte 11(39), 62-78. Recuperado de https://doi.org/10.5232/ricyde2015.03906

Búa, N., Rodríguez, A., García, G. (2013). Perfil funcional y morfológico en jugadores de fútbol amateur de Mendoza, Argentina. Apunts. Medicina de l'Esport, 48(179), 89-96. Recuperado de https://doi.org/10.1016/j.apunts.2012.07.001

Calahorro, F., Zagalaz, M., Lara, A., Torres, G. (2012). Análisis de la condición física en jóvenes jugadores de fútbol en función de la categoría de formación y del puesto específico. Revista-Apunts Educació Física I Esports, 109, 54-62. Recuperado de https://doi. org/10.5672/apunts.2014-0983.es.(2012/3).109.05

Cardenal, J., Quintero, E. (2015). Evaluación del VO2max y composición corporal en futbolistas pre juveniles de la academia de fútbol Comfenalco. (Tesis de Licenciatura). Universidad Santo Tomás, Bucaramanga. Recuperado de http://porticus.usantotomas.edu. co/bitstream/11634/900/1/2015-CardenalDaza,JonathanEduardo-Tesis.pdf

Carrasco, J., Calahorro, F., Lara, A., Torres, G. (2014). Efectos de un Programa de Entrenamiento de Fútbol sobre la Condición Física en Jugadores Jóvenes. Kronos, 13(1). Recuperado de http://g-se.com/es/journals/kronos/articulos/efectos-de-un-programa-de-entrenamientode-futbol-sobre-la-condicion-fisica-en-jugadores-jovenes-1699

Castellanos, G., Sánchez, A., González, M., Carvajal, W. (2011). Capacidad De Trabajo Físico En Alumnos Del Grupo De Combate De Los Centros De Perfeccionamiento Deportivo 
Sideportes De Guatemala. Rev. Cub. Med. Dep. \& Cul. Fís. (7)1. Recuperado de http://www. imd.inder.cu/adjuntos/article/277/Capacidad $\% 20 \mathrm{de} \% 20$ trabajo $\% 20 \mathrm{f} \% \mathrm{C} 3 \% \mathrm{ADsic0} \% 20$ en $\% 20$ alumnos $\% 20$ del $\% 20$ grupo $\% 20 \mathrm{de} \% 20$ combate.pdf

Cornejo, J. (2016). Perfil antropométrico del jugador de fútbol categoría sub 16 de O’Higgins de Rancagua. Revista Ciencias de la Actividad Física, 16(2). Recuperado de https://revistacaf. com/ojs/index.php/RCAF/article/view/11

Fernández, J., Kazarez, M., Agazzi, B., Albin, S. (2014). Evaluación Antropométrica según Posición de Juego de Jugadores Profesionales del fútbol Uruguayo. Revista Enfermería Cuidados Humanizados, 3(2). Recuperado de http://revistas.ucu.edu.uy/index.php/ enfermeriacuidadoshumanizados/article/view/566

Frazilli, E. de Arruda, M., Mariano, T., Cossio, M. (2011). Correlación entre fuerza explosiva y velocidad en jóvenes. Biomecánica, 19(1), 19-24. Recuperado de https://upcommons.upc. edu/bitstream/handle/2099/12308/19-24.pdf

Henríquez, C., Báez, E., Ramírez, R., Cañas, R. (2013). Perfil somatotípico del futbolista profesional chileno. International Journal of Morphology, 31(1), 225-230. Recuperado de http://www.scielo.cl/scielo.php?pid=S0717-95022013000100037\&script=sci arttext https:// doi.org/10.4067/S0717-95022013000100037

García, F., Peña, J. (2016). Efectos de 8 semanas de entrenamiento pliométrico y entrenamiento resistido mediante trineo en el rendimiento de salto vertical y esprint en futbolistas amateurs. Kronos, 15(2). Recuperado de http://g-se.com/es/entrenamiento-de-la-velocidad-y-agilidad/articulos/efectosde-8-semanas-de-entrenamiento-pliometrico-y-entrenamiento-resistido-mediante-trineo-en-elrendimiento-de-salto-vertical-y-esprint-en-futbolistas-amateurs-2192

García, F., Ruiz, A., Latorre, P. (2015). Influencia del puesto específico en la potencia y agilidad de jóvenes futbolistas. Retos: Nuevas tendencias en Educación Física, Deportes y Recreación, 27, 58-61. Recuperado de https://dialnet.unirioja.es/descarga/articulo/5407716.pdf

Gil, J., Verdoy, P. (2011). Caracterización de Deportistas Universitarios De Fútbol Y Baloncesto: Antropometría Y Composición Corporal. Revista de Ciencias del Deporte, 7(1), 39-51. Recuperado de http://e-balonmano.com/ojs/index.php/revista/article/view/65/57

González, G., Campos, M., Romero, S. (2014). Análisis de la influencia de la evaluación del rendimiento en jugadores de un equipo de fútbol. Retos. Nuevas tendencias en Educación Física, Deporte y Recreación, 25, 85-89. Recuperado de https://dialnet.unirioja.es/descarga/ articulo/4554984.pdf

Iglesias, P., Grijota, F., Crespo, M., Llerena, F., Muñoz, D. (2013). Efectos de la práctica de fútbol sobre la composición corporal, en jóvenes deportistas entrenados y no entrenados. 
European Journal of Human Movement, 31, 135-146. Recuperado de https://dialnet. unirioja.es/servlet/articulo?codigo $=4775818$

Jorquera, C., Rodríguez, F., Torrealba, M., Campos, J., Gracia, N., \& Holway, F. (2013). Características antropométricas de futbolistas profesionales chilenos. International Journal of Morphology, 31(2), 609-614. Recuperado de https://doi.org/10.4067/S0717-95022013000200042

Jorquera, C., Rodríguez, F., Torrealba, M., Barraza, F. (2012). Composición Corporal y Somatotipo de Futbolistas Chilenos Juveniles Sub 16 y Sub 17. International Journal of Morphology, 30(1), 247-252. Recuperado de https://doi.org/10.4067/S0717-95022012000100044

Kalentić, Ž., Golik, D., Doder, D., Sudarov, N., \& Jovančević, V. (2012). Differences in some morphological characteristics and thigh muscle force of bounce leg between football players and goalkeepers. Exercise and Quality of Life, 4(1), 33-39. Recuperado de http:// scindeks.ceon.rs/article.aspx?artid=1821-34801201033K

Le Gall, F. Carling, C., Williams, M. \& Reilly, T. (2010). Anthropometric and Fitness characteristics of international, professional and amateur male graduate soccer player from elite youth academy. Journal of Science and Medicine in Sport, 13(1), 90-95. Recuperado de http://dx.doi.org/10.1016/j.jsams.2008.07.004.

Mariano, T., de Arruda, M., Pascoal, E. (2010). Jovens Futebolistas: Contribuição Da Maturação E Variáveis Antropométricas No Desenvolvimento Da Força Explosiva E Velocidade Em Púberes E Pós-Púberesdurante Três Meses De Treinamento. Conexões, 8(2). Recuperado de http:/periodicos.sbu.unicamp.br/ojs/index.php/conexoes/article/download/8637744/5435

Maureira, F., Bahamondes, V., Jesam, B, López, R. (2015). Tiempo De Reacción Y Tiempo De Ejección En Arqueros De Fútbol Categoría Sub-15. El Físico de Chile, 53-61. Recuperado de https://dialnet.unirioja.es/descarga/articulo/4347424.pdf

Méndez, E., Márquez, J., Castro, C. (2011). El trabajo de fuerza en el desarrollo de la potencia en futbolistas de las divisiones menores de un equipo profesional de fútbol. Revista médica Universidad de Antioquía, 20(2), 27- 43. Recuperado de https://aprendeenlinea.udea.edu. co/revistas/index.php/iatreia/article/view/4393

Morgans, R., Orme, P., Anderson, L., Drust, B. (2014). Principles and practices of training for soccer. Journal of Sport and Health Science, 3(4), 251-257. Recuperado de https://doi. org/10.1016/j.jshs.2014.07.002

Hernández-Mosqueira, C., Fernandes, S., Fernandes, J., Retamales, F., Ibarra, J., Vasquez, D., Valenzuela, R. (2013). Descripción de la composición corporal y somatotipo de fútbolistas sub 18, en función de la posición en el campo. European Journal of Human Movement, (31), 147-158. Recuperado de http://www.redalyc.org/articulo.oa?id=274229586010 
Muñoz, V. (2015). Cuantificación y análisis de las demandas físicas y respuestas fisiológicas en el fútbol juvenil durante entrenamientos y competición, y su relación con el perfil condicional. (Tesis de doctorado). Universidad de Castilla la Mancha, Toledo. Recuperado de https://ruidera.uclm.es/xmlui/bitstream/handle/10578/7174/TESIS\%20 Mu\%C3\%B1oz $\% 20$ Fern $\% \mathrm{C} 3 \% \mathrm{~A} 1$ ndez $\% 20$ Arroyo.pdf? sequence $=1 \&$ is Allowed $=\mathrm{y}$

Oliveros, D. (2015). Algunos Factores Biomécanicos de la carrera en Ascenso. Lúdica Pedagógica, 1(3). Recuperado de http://www.oei.es/historico/n2822.htm

Pazo, C., Sáenz, P., Fradua, L. (2012). Influencia del contexto deportivo en la formación de los futbolistas de la selección española de fútbol. Revista de Psicología del Deporte. 21(2). 291-299. Recuperado de http://www.rpd-online.com/article/view/874/857

Portella, D. L., De Arruda, M., Cossio, M. A. (2011). Valoración del rendimiento físico de jóvenes futbolistas en función de la edad cronológica. Educació Física i Esports, (106), 42. Recuperado de https://doi.org/10.5672/apunts.2014-0983.es.(2011/4).106.05

Prieto, Y., García, J. (2013). Efectos de un entrenamiento específico de potencia aplicado a futbolistas juveniles para la mejora de la velocidad con cambio de dirección. European Journal of Human Movement, (31), 17-36. Recuperado de https://recyt.fecyt.es/index.php/ ejhm/article/view/56401

Ramos, S. Aguirre, H., Gutiérrez, L. (2015). Deportistas Escolares Centroamericanos: Proceso de identificación y detección de talentos. Revista Impetus, 9(2), 23-33. Recuperado de http://revistaimpetus.unillanos.edu.co/impetus/index.php/Imp1/article/view/138/109

Ruiz, A., Romero, R., Fernández, A., Morcillo, J., Mariscal, M. (2015). Estimación del gasto de energía en un partido amistoso de jugadores de fútbol de primera división. Revista Andaluza de Medicina del Deporte, 8(4), 176-176. Recuperado de https://doi.org/10.1016/j.ramd.2015.01.006

Salazar, J., Romo, J., Sánchez, A., Moreno, P., Pineda, L. y Carranza, I. (2017). Diferencias en el OBLA en jugadoras de fútbol en relación a su posición en el campo de juego (OBLA' s differences in soccer players according to their field position). Retos, (32), 58-61. Recuperado de https://recyt.fecyt.es/index.php/retos/article/view/48937

Sánchez, B., Ureña, P., Salas, J., Blanco, L., Araya, F. (2011). Perfil Antropométrico y Fisiológico en Futbolistas de Élite Costarricenses según Posición de Juego. PubliCE Standard. Recuperado de http://g-se.com/es/antropometria/articulos/perfil-antropometrico-yfisiologico-en-futbolistas-de-lite-costarricenses-segun-posicion-de-juego-1382

Sánchez, B., Araya, F., Blanco, L., Crespo, C. (2016). Comparación de dos métodos para medir la composición corporal de futbolistas profesionales costarricenses. Revista MHSalud, 12(2), 1-13. Recuperado de https://doi.org/10.15359/mhs.12-2.1 
Sánchez, P., Pérez, F., Coco, C., Ruiz, F., Marín, D. (2013). Efectos de la práctica de fútbol sobre la composición corporal en jóvenes deportistas entrenados y no entrenados. European Journal of Human Movement, 31, 135-146. Recuperado de https://recyt.fecyt.es/index.php/ ejhm/article/view/56408

Sinivas, M., Pérez, A., Álvarez, I., Bores, A., Ramos, D., Rubio, J., Cerrato, D. 2015. Influencia De La Composición Corporal Sobre El Rendimiento En Salto Vertical Dependiendo De La Categoría De La Formación Y La Demarcación En Futbolistas. Revista Nutrición Hospitalaria, 32(1), 299307. Recuperado de http://www.aulamedica.es/gdcr/index.php/nh/article/view/8876/pdf 8199

Soarez, H., Fragoso, I., Massuça, L., Barrigas, C. (2012). Impacto de la maduración y de los puestos específicos en la condición física en jóvenes futbolistas. Apunts. Medicina de l'Esport, 47(174), 73-81. Recuperado de http://appswl.elsevier.es/watermark/ ctl servlet? $\mathrm{f}=10 \&$ pident articulo $=90142469 \&$ pident usuario $=0$ \&pcontactid $=\&$ pident revista $=277 \&$ ty $=161 \&$ accion $=L \&$ origen $=$ bronco $\% 20 \& w e b=w w w$. apunts. org\&lan=es\&fichero $=277 \mathrm{v} 47 \mathrm{n} 174 \mathrm{a} 90142469 \mathrm{pdf001}$.pdf\&anuncioPdf $=$ ERROR publi_pdf https://doi.org/10.1016/j.apunts.2011.08.004

Tissue, C. (2014). Modificación del tejido adiposo y el somatotipo en futbolistas amateurs y adolescents durante el período precompetitivo. Journal of Sport and Health Research, 6(2), 139-150. Recuperado de http://www.journalshr.com/papers/Vol\%206_N\%202/V06_2_3.pdf

Toro, E. y Pelaez, E. (2014). Caracterización antropométrica, motriz y funcional de jugadores de futbol de 14 y 15 años en la Escuela de Formación Zurety de la ciudad de Cali. (Tesis de Licenciatura). Universidad del Valle, Cali. Recuperado de http://bibliotecadigital.univalle. edu.co/bitstream/10893/7668/1/3484-0473492.pdf

Ziv, G., Lidor, R. (2011). Physical characteristics, physiological attributes, and on-field performances of soccer goalkeepers. International Journal of Sports Physiology and Performance, 6(4), 509-524. Recuperado de https://doi.org/10.1123/ijspp.6.4.509

Zubeldía, G. (2010). Características físicas y antropométricas correspondientes a las divisiones del fútbol juvenil del club Atlético Lanus. PubliCE Standard. Recuperado de http://g-se.com/ es/antropometria/articulos/caracteristicas-fisicas-y-antropometricas-correspondiente-alas-divisiones-del-futbol-juvenil-del-club-atletico-lanus-898

Fecha de recibo:8 de setiembre del 2016 Fecha de corrección: 26 de abril del 2016

Fecha de aceptación: 16 de mayo del 2017

Fecha de publicación: 9 de junio del 2017 